\title{
Interfacial thermal resistance between the graphene-coated copper and liquid water
}

\author{
An Truong Pham ${ }^{a}$, Murat Barisik ${ }^{\mathrm{b}}$, BoHung Kim ${ }^{\mathrm{c}, *}$ \\ a Department of Mechanical Engineering and Materials Science, Duke University, Box 90300 Hudson Hall, Durham, NC 27708, USA \\ ${ }^{b}$ Department of Mechanical Engineering, Izmir Institute of Technology, Urla 35430, Izmir, Turkey \\ ' School of Mechanical Engineering, University of Ulsan, Daehak-ro 93, Namgu, Ulsan, South Korea
}

\section{A R T I C L E I N F O}

\section{Article history:}

Received 21 May 2014

Received in revised form 12 February 2016

Accepted 14 February 2016

Available online 27 February 2016

\section{Keywords:}

Molecular dynamics simulations

Kapitza length

Graphene

Nano-composite

\begin{abstract}
A B S T R A C T
The thermal coupling at water-solid interfaces is a key factor in controlling thermal resistance and the performance of nanoscale devices. This is especially important across the recently engineered nano-composite structures composed of a graphene-coated-metal surface. In this paper, a series of molecular dynamics simulations were conducted to investigate Kapitza length at the interface of liquid water and nano-composite surfaces of graphene-coated-Cu(111). We found that Kapitza length gradually increased and converged to the value measured on pure graphite surface with the increase of the number of graphene layers inserted on the Cu surface. Different than the earlier hypothesis on the "transparency of graphene," the Kapitza length at the interface of mono-layer graphene coated $\mathrm{Cu}$ and water was found to be 2.5 times larger than the value of bare $\mathrm{Cu}$ surface. This drastic change of thermal resistance with the additional of a single graphene is validated by the surface energy calculations indicating that the mono-layer graphene allows only $\sim 18 \%$ van der Waals energy of underneath $\mathrm{Cu}$ to transmit. We introduced an "overall interaction strength" value for the nano-composites based the quantitative contribution of pair interaction potentials of each material with water into the total surface energy in each case. Similar to earlier studies, results revealed that Kapitza length shows exponentially variation as a function of the estimated interaction strength of the nano-composite surfaces. The effect of $\mathrm{Cu} /$ graphene coupling on thermal behavior between the nano-composite with water was characterized. The Kapitza length was found to decrease significantly with increased $\mathrm{Cu} / g r a p h e n e$ strength in the case of weak coupling, while this behavior becomes negligible with strong coupling of $\mathrm{Cu}$ and graphene.
\end{abstract}

(c) 2016 Elsevier Ltd. All rights reserved.

\section{Introduction}

Progress in the area of integrated circuit technologies has led to the rapid growth of semiconductor industry. Currently, silicon and silicon-based materials used in semiconductors have entered the realm of sub-20 nm dimensions in order to meet the demand for low-power, multifunctional electronic chips with high performance and large data storage capabilities [1]. While the reduction in size smaller than $10 \mathrm{~nm}$ is a challenge for existing technology [2], the future generation of devices will soon have lowdimensional physics founded by a single or couple of molecular structures. In such cases, graphene has been considered as a key material with potential to overcome the current limitations by providing extraordinary properties such as: high mechanical strength and flexibility [3]; ultrahigh electron mobility [4-5]; and high ther-

\footnotetext{
* Corresponding author.

E-mail address: bohungk@ulsan.ac.kr (B. Kim).
}

mal conductivity [6]. On such direction, one of the main technological accomplishments is the development of various approaches to synthesize graphene on metal substrates [7]. Graphene with a single atom thickness of carbon acts as an ultrathin oxidationresistant coating with minimal changes in the metal properties [8].

Heat removal is a challenge that restricts the use of grapheneassisted surface coatings in nanoscale devices. The performance and reliability of these devices strongly depends on the efficient dissipation of heat from solid substrates to the surrounding environment or coolant systems. Therefore, understanding the effects of mono- or multi-layers graphene coatings onto thermal transport behavior at the interfaces of graphene-coated-metal structures that neighbor either solid or fluid is critical to thermal management design for new technologies. Recently, the interfacial thermal resistance, known as the Kapitza resistance [9] between liquid and metal/graphene nano-composite has attracted much attention with the objective of obtaining a deeper understanding of heat transport across the interface. For instance, the interfacial thermal 
conductance and interfacial phonon transmission coefficients of nano-composite consisting of several layers of graphene sandwiched between silicon crystals were investigated in the study of Shen et al. [10]. Interfacial thermal resistance was found to depend not only on the strictly defined interface properties, but also to associate with the near interface-region when the confined graphene layer is strongly coupled with relatively neighboring materials [11]. Mao et al. [12] used first-principle calculations and an atomistic phonon transport model based on Landauer formalism to demonstrate the strong dependence of thermal transfer on the interlayer separation and stronger bonding of several solid materials with graphene. Chang et al. [13] found a strong dependence of interfacial thermal conductance on the number of graphene layers confined between metal phases. Despite multiple successful studies on metal/graphene systems, there are still very few data pertaining to the thermal behavior of graphene-coated-metal/liquid interfaces. Studies covering liquid water are particularly crucial due to the common use of water in numerous cooling applications for Micro and Nano Electro Mechanical System (MEMS and NEMS).

Over the past decade, molecular dynamics simulations has emerged as a powerful tool to investigate thermal resistance at the interface of solids and water, providing valuable insight into interfacial energy transfer mechanisms. In particular, Kapitza resistance at solid/liquid interfaces are found to depend significantly on the strength of the solid/liquid interaction [14-17], bulk liquid pressure and wettability of solid surfaces [18], the surface temperature [19-22], the interaction energy per unit area of solid/water contact surfaces [23], and hydrophilic headgroups or selfassembly of mono-layers with different chain lengths assigned onto solid surfaces [24]. With extraordinary properties of graphene, the understanding of interfacial thermal resistance at nano-composite surfaces that are composed of mono- or multilayers of graphene on metal surface and liquid water, is expected to play an important role towards the development of heat transfer and microfluidics devices.

For nano-composites of graphene-coated-metal substrates, water contact angle measurements were employed to characterize the surface energy between liquid water and the nanocomposite surfaces [25-27]. The simple Lorentz-Berthelot mixing rule [28] is not a reliable approach to calculate the interaction parameters of non-identical molecules. Hence, the interaction strength between metal/water and graphene/water were parameterized to match the experimentally measured contact angle of the corresponding substrate. This can provide a correct physical behavior for thermal vibrations between solid and liquid, and accurate modeling of the molecular heat transfer mechanisms at the interfaces [29]. The interaction strength between metal substrates and graphene is another important factor that influences the heat transfer across the interfaces of graphene-coatedmetal/water systems. For instance, Hopkins et al. [30] found that interfacial chemical bonding of both the phonon flux and the vibrational mismatch has significant influence on the thermal boundary conductance of the metal/graphene. Chen et al. [31] reported that out-of-plane acoustic phonon modes of graphene have significantly changed due to the strong coupled interaction strength between the metal substrate and graphene. In order to simulate the correct behavior of the phase transitions of partially confined copper nanowires inside carbon nanotubes, Guo et al. [32] obtained the Lennard-Jones (LJ) parameter of $\mathrm{Cu}-\mathrm{C}$ using the mixing rule, and verified that value by first-principle density functional theory (DFT) calculations. Despite the parameter being suitable validated and universally approved in the research of heat transfer performance of metal coated interfaces [13,33], the $14 \%$ discrepancy of the binding energies calculated by MD simulations and the DFT approach is not the most suitable for $\mathrm{Cu}-\mathrm{C}$ interaction strength [32]. Therefore, the dependence of interfacial thermal resistance on metal/graphene interaction strength needs to be investigated further.

In this work, a series of non-equilibrium molecular dynamics simulations were conducted to investigate the effects of graphene coatings on the interfacial thermal resistance between the graphene-coated-Cu(111) surface and liquid water. We utilized calibrated copper/water and graphene/water interaction strength parameters, which captured the wetting behaviors of the copper and graphite with liquid water. The copper/graphene coupling was also varied to characterize the impact of metal-graphene bonding on interfacial thermal transfer. The Kapitza lengths were measured while the number of graphene layers was varied from 1 to 5 in order to create different surface coating cases. The interfacial energies per unit area of nano-composite having different number of graphene layers with liquid water were theoretically calculated to predict the surface energy of each nano-composite case. We defined an overall interaction strength value between the nano-composite and water based on the quantitative contribution of $\mathrm{Cu} /$ water and graphene/water pair interaction potential energies into the calculated surface energy of the nanocomposite for each different case. The variation of overall nanocomposite/water interaction strength values were employed to characterize the variation of Kapitza length using our earlier exponential dependence theory.

\section{Molecular structure and simulation details}

A schematic diagram of the simulation structure is shown in Fig. 1. Liquid water was confined between two parallel graphenecoated-Cu(111) surfaces in which the graphene-coated layer faces the fluid. The simulation dimensions were $30.95 \AA$ in the $x$-direction, $33.28 \AA$ in the $y$-direction, and the length of the $z$-direction was adjusted depending on the number of grapheneassist surface coatings. The copper formed by 13 layers of $\mathrm{Cu}$ (111) was $25.01 \AA$ thick in the $z$-direction with an interlayer spacing of $2.084 \AA$. The number of graphene layers coated on the $\mathrm{Cu}$ (111) surfaces was from 1 to 5 and the interlayer spacing between the graphene-graphene and $\mathrm{Cu}$-graphene was 3.4 $\AA$. The liquid density in the middle of the simulation box was set to be close to saturated liquid water density at the corresponding temperature and pressure. Specifically, 2400 water molecules were contained in a volume of $30.95 \AA \times 33.28 \AA \times 70 \AA$, matching the saturated liquid water density of $\rho=0.9957 \mathrm{~g} / \mathrm{cm}^{3}$ at temperature of $303 \mathrm{~K}$ and pressure of $4.5 \mathrm{kPa}$.

$\mathrm{A} \mathrm{Cu}(111)$ surface structure was chosen as a solid base in this study as it was the best match with graphene [34] and the growth of graphene on $\mathrm{Cu}(111)$ was achieved in an ultrahigh vacuum chamber previously [35]. In particular, the lattice constant of $\mathrm{Cu}$ (111) is $a_{\mathrm{Cu}}=2.552 \AA$, while graphene's well-known honeycomb lattice structure matches the triangle-shaped lattice of the $\mathrm{Cu}$ (111) surface shown in Fig. 1(b and c). The present lattice constant of graphene is $2.552 \AA$, which is approximately $3.74 \%$ higher than its original value of $a_{\mathrm{Gr}}=2.46 \AA$. The resulting graphene lattice constant has been shown to be a stable atomic structure at the graphene-copper interface, free from any defects or breakage of graphene sheets periodic condition [34,36].

In order to conduct non-equilibrium molecular dynamics simulations of heat transfer through these systems, the outermost layers of both sides of the simulation domains were fixed to their original positions to maintain a system of constant volume. Thermostats were placed at the outermost second and third layers of both solid walls. That means the thermostat reservoirs were small groups of solid atoms next to the outermost layers on the left and right hand side of the system corresponding to hot and cold thermostat regions, respectively. The remains of solid atoms and liquid 


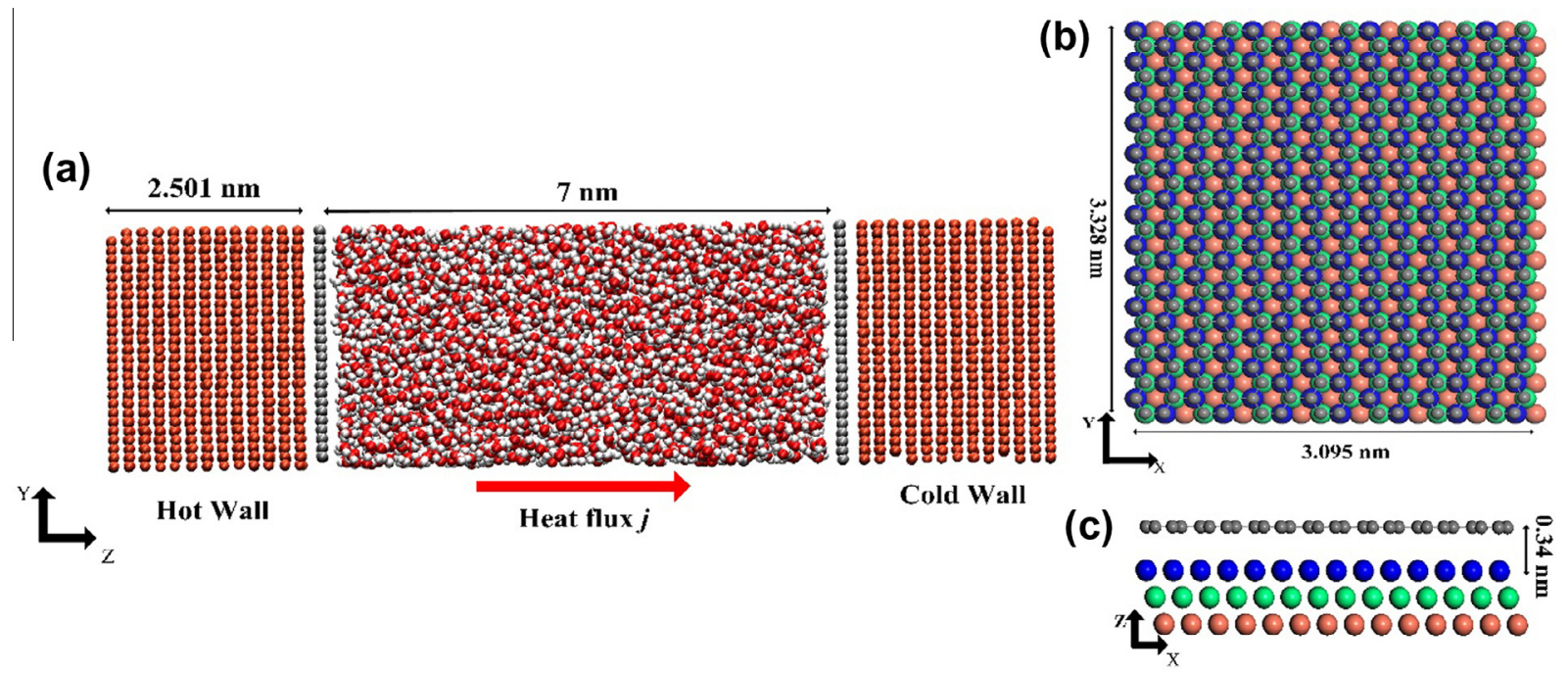

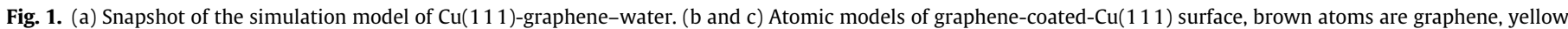

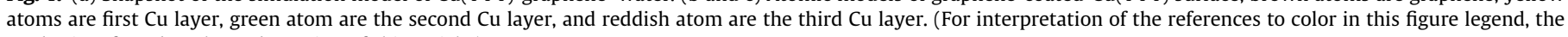
reader is referred to the web version of this article.)

water in the system were free to move without a thermostat applied when heat transfer occurred. Periodic boundary conditions were enforced in $x$ - and $y$-directions.

In this study, for the purpose of simplicity and computational efficiency, the simple point charge (SPC/E) model was chosen, which can be described as an effective rigid pair potentials composed of Lennard-Jones (LJ) and Coulombic terms [37]. This water model has three interaction sites, corresponding to the three atoms of the water molecules. Each atom was assigned a point charge to model the long-range Coulombic interactions, and the oxygen atom also exhibited $\mathrm{LJ}$ potential to model van der Waals (vdW) forces. In particular, the partial charges $q_{O}=-0.8476 e$ and $q_{H}=0.4238 e$ were assigned to oxygen and hydrogen atoms to model Coulombic interactions. Long-range electrostatic forces were calculated by a particle-particle, particle-mesh (PPPM) solver [28]. The harmonic O- $\mathrm{H}$ bond length of $0.1 \mathrm{~nm}$ and the $\mathrm{H}-\mathrm{O}-\mathrm{H}$ angle of $109.47^{\circ}$ were kept rigid using the SHAKE algorithm [38]. LJ interactions were calculated between the wall molecules and the oxygen atoms of the liquid water. We use the truncated LJ (12-6) potential to model the vdW interactions given as

$U_{\text {truncated }}\left(r_{i j}\right)=4 \varepsilon\left[\left(\left(\frac{\sigma}{r_{i j}}\right)^{12}-\left(\frac{\sigma}{r_{i j}}\right)^{6}\right)-\left(\left(\frac{\sigma}{r_{c}}\right)^{12}-\left(\frac{\sigma}{r_{c}}\right)^{6}\right)\right]$,

where $\varepsilon$ is the binding potential between solid and liquid, $r_{i j}$ is the intermolecular distance, $\sigma$ is the finite molecular distance at which interatomic potential is zero, and $r_{c}$ is the cut-off distance.

Intermolecular forces between the $\mathrm{Cu}$ molecules were modeled by the embedded atom method (EAM) [39], which describes the total energy of a metal by calculating the embedding energy as a function of the atomic electron density. In order to model the interatomic interactions of $\mathrm{C}-\mathrm{C}$ in the graphene sheets, the adaptive intermolecular reactive empirical bond-order (AIREBO) potential [40] was employed. The AIREBO potential is widely used to calculate the potential energy of covalent bonds and interatomic forces in condensed-phase hydrocarbon systems such as graphite. In our study, the calibration of interaction strength and molecular distance parameters of solid with liquid water were conducted by measuring the contact angle of 2000 water molecules droplet on pure $\mathrm{Cu}$ and 5-layers of graphene coated $\mathrm{Cu}$ substrates. Specifically, we used Lorentz-Berthelot [28] mixing rules to define the distance parameter between $\mathrm{Cu}$ and water as $\sigma_{\mathrm{Cu}-\mathrm{O}}=\frac{\sigma_{\mathrm{Cu}-\mathrm{Cu}}+\sigma_{\mathrm{O}-\mathrm{O}}}{2}=2.751 \AA$, where $\sigma_{\mathrm{Cu}-\mathrm{Cu}}=2.337 \AA[41]$ and $\sigma_{\mathrm{O}-\mathrm{O}}=3.166 \AA$. The interaction strength was set as $\varepsilon_{\mathrm{Cu}-\mathrm{O}}=0.00737 \mathrm{eV}$, which reproduced the microscopic water droplet contact angle of $86.1^{\circ}$ on Cu substrate. The parameters between graphite and water were $\varepsilon_{\mathrm{C}-\mathrm{O}}=$ $0.00412 \mathrm{eV}, \sigma_{\mathrm{C}-\mathrm{O}}=3.19 \AA$ which also recovered the corresponding microscopic contact angle of $96.1^{\circ}$. The measured contact angle values of our study were found in a good agreement with reported values from the studies of Rafiee et al. [25] for bare $\mathrm{Cu}\left(85.9^{\circ}\right)$, and Werder et al. [42] for graphite $\left(95.3^{\circ}\right)$, respectively. LJ potentials were also used to model the interactions between metal and graphene sheets. We used the parameters reported by Guo et al. [32] which were $\varepsilon_{\mathrm{Cu}-\mathrm{C}}=0.02578 \mathrm{eV}$ and $\sigma_{\mathrm{Cu}-\mathrm{C}}=3.0825 \AA$. The details of molecular parameters used are given in Table 1.

The Irving-Kirkwood (I-K) expression was utilized to calculate the heat flux vector for an $\mathrm{N}$ particles system using unity differential operator approximation as follows $[43,44]$ :

$$
\begin{aligned}
J_{k}= & \frac{1}{\mathrm{Vol}}\left\langle\sum_{i}^{N} v_{k}^{i}\left(E^{i}+U^{i}\right)-\sum_{i}^{N} S_{k}^{i} v_{k}^{i}\right\rangle, \\
E^{i}= & \frac{1}{2} m^{i}\left(\left(v_{x}^{i}\right)^{2}+\left(v_{y}^{i}\right)^{2}+\left(v_{z}^{i}\right)^{2}\right), \\
S_{k}^{i}=- & \left(\frac{1}{2} \sum_{n=1}^{N_{p}}\left(r_{k}^{1} F_{k}^{1}+r_{k}^{2} F_{k}^{2}\right)+\frac{1}{2} \sum_{n=1}^{N_{b}}\left(r_{k}^{1} F_{k}^{1}+r_{k}^{2} F_{k}^{2}\right)\right. \\
& \left.+\frac{1}{3} \sum_{n=1}^{N_{a}}\left(r_{k}^{1} F_{k}^{1}+r_{k}^{2} F_{k}^{2}+r_{k}^{3} F_{k}^{3}\right)+\operatorname{Kspace}\left(r_{k}^{i}, F_{k}^{i}\right)+\sum_{n=1}^{N_{f}} r_{k}^{i} F_{k}^{i}\right),
\end{aligned}
$$

where the first term on the right hand side of Eq. (2) represents the kinetic $\left(E^{i}\right)$ and potential $\left(U^{i}\right)$ energies carried by particle $i$; and the second term is the energy transfer to particle $i$ by force interactions

Table 1

Interaction parameters were used for simulation domains.

\begin{tabular}{llll}
\hline Interaction & $\sigma(\AA)$ & $\varepsilon(\mathrm{eV})$ & $q(\mathrm{e})$ \\
\hline $\mathrm{H}-\mathrm{H}$ & 0 & 0 & 0.4238 \\
$\mathrm{O}-\mathrm{O}$ & 3.166 & 0.006739 & -0.8476 \\
$\mathrm{Cu}-\mathrm{O}$ & 2.75185 & 0.00737 & 0 \\
$\mathrm{Cu}-\mathrm{C}$ & 3.0825 & 0.02578 & 0 \\
$\mathrm{C}-\mathrm{O}$ & 3.19 & 0.00412 & 0 \\
\hline
\end{tabular}


with the surrounding particles which is represented in terms of virial component of per-atom stress tensor $\left(S_{k}^{i}\right)$ and velocity of particle $\left(v_{k}^{i}\right)$. Specifically, the kinetic energy of particle is expressed in Eq. (3), while potential energy is extracted from Eq. (1). Eq. (4) represents the stress tensor for atom $i$ in the axes $k$ of the Cartesian coordinate system, excluding kinetic energy term as we described in our previous study [18]. The contribution of intermolecular forces were extracted from viral of atomistic stress tensor as the first term is a pairwise energy contribution where $n$ loops over the $N_{p}$ neighbors of atom $i, r_{k}^{1}$ and $r_{k}^{2}$ are the positions, and $F_{k}^{1}$ and $F_{k}^{2}$ are the forces of the two atoms in the pair-wise interaction; the second and third terms are bond and angle contribution on atom $i$; the Kspace term is the contribution from long-range Coulombic interactions for PPPM solver; and finally, fifth term is the SHAKE internal constraint force to particle $i$. Therefore, an overall heat flux was calculated in the water volume using Eq. (2) by considering the contributions of each atom within a water molecule.

Simulations began with an NVT (constant number of molecules, constant volume, and constant temperature) ensemble to establish system equilibrium in the first 2 ns. In this state, the MaxwellBoltzmann velocity distribution was used as the initial velocity for all molecules, and a Nose-Hoover thermostat maintained system temperature of $320 \mathrm{~K}$. In order to induce heat flux across the simulation domain, different temperatures were assigned to hot and cold reservoirs using Langevin thermostat, while the remaining solid lattices and fluid were maintained at NVE (constant number of molecules, constant volume, and constant energy) ensemble. In this second state, heat transfer simulations were performed for $6 \mathrm{~ns}$, with the first $2 \mathrm{~ns}$ allowed for the system to reach a steady state in the presence of applied heat, and the rest for averaging system's temperature distributions and heat flux. The simulation time step was set as 1.0 femtosecond (fs). All simulations were performed using LAMMPS [45].

\section{Results and discussion}

\subsection{Effect of number of graphene layers on Kapitza length}

In order to understand the effect of number of the graphene layers to the near surface liquid structures, the local density distributions were studied by dividing the simulation domains into slab bins with a bin size of $0.2 \AA$ in the $z$-direction. Despite the fact that the chosen small bin size could induce significant fluctuations in local temperature calculations near solid surfaces [18], small size bin is an advantage to define the location of surface and the separation distance from solid to liquid region accurately. Density distributions of liquid water near the hot surface are given in Fig. 2, where the yellow shade color indicates the solid region. Water density exhibited oscillatory behavior over several atomic distances from the solid surface. This oscillation gradually converged to a constant value of $1 \mathrm{~g} / \mathrm{cm}^{3}$ in the bulk region of the channel. In the density layering found on the interface, liquid molecules mimicked the solid lattice structure under the dominant surface forces in this region. The increase in water density is governed by the surface energies between water and solid substrates. In case of graphene-coated-Cu surface, graphene separates $\mathrm{Cu}$ from liquid molecules; and hence, graphene mostly contributes to surface energy despite the stronger depth of potential well of $\mathrm{Cu}$ with liquid water. The change of water density with the addition of graphene layer can be understood by comparing the $\mathrm{Cu}-\mathrm{O}$ and $\mathrm{C}-\mathrm{O}$ interaction strengths given in Table 1 . Specifically, the interaction potential ratio of $\mathrm{Cu}-\mathrm{O}$ to $\mathrm{O}-\mathrm{O}$ molecules is $\varepsilon_{\mathrm{Cu}-\mathrm{O}} / \varepsilon_{\mathrm{O}-\mathrm{O}} \approx 1.094$, while this value for $\mathrm{C}-\mathrm{O}$ and $\mathrm{O}-\mathrm{O}$ is $\varepsilon_{\mathrm{C}-\mathrm{O}} / \varepsilon_{\mathrm{O}-\mathrm{O}} \approx 0.611$. As a result, the interaction strength between pure $\mathrm{Cu}$ with liquid water is stronger than the case of coating it with graphene layers. Stronger

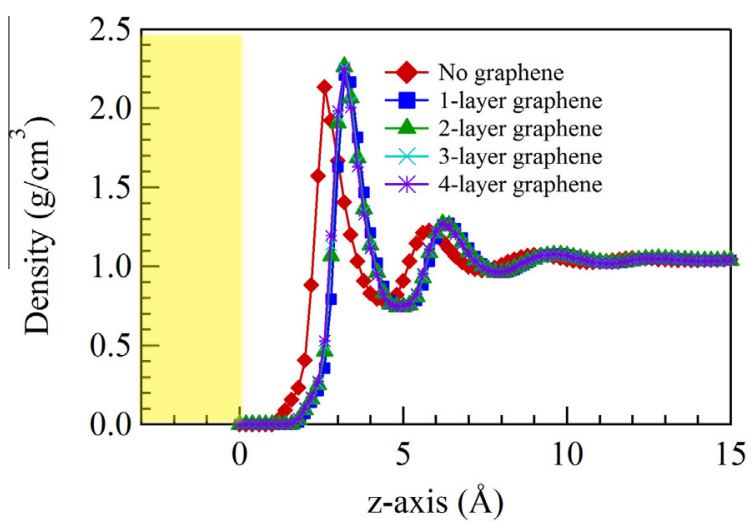

Fig. 2. Density distributions of liquid water near solid surfaces of pure $\mathrm{Cu}$ and several graphene layers coated on $\mathrm{Cu}$. The yellow shade indicates solid region. (For interpretation of the references to color in this figure legend, the reader is referred to the web version of this article.)

interaction potential also created smaller depletion region of liquid water next to $\mathrm{Cu}$ than the depletion length next to the graphene. In particular, the first liquid water density maxima is $2.5 \AA$ away from pure $\mathrm{Cu}$ surface, while the location of this maxima is $3.2 \AA$ away from graphene. As discussed in our previous study in details [18], density depletion length can be considered as a quantity to represent the thermal coupling of solid with water as a function of surface properties.

Despite the significant change of water density with the addition of the first graphene layer, graphene-coated-Cu surface density profiles found similar to each other. The density profile remains unchanged by the addition of more graphene layers from 1 to 5 . This reveals that the interactions between graphene coating and water dominates in graphene/Cu nano-structure; which allows only partial transmission of the energy from the $\mathrm{Cu}$ to the liquid domain. This result can be considered as the first evidence of the behavior observed here as the partial transparency. This observation challenged the wetting transparency of graphene observed in Ref. [25], which presents a negligible change of water structure next to solid substrate with the first graphene coating, and it continued changing by the increase of number of graphene up to three layers. We will further investigate this result by studying the surface energy and the interfacial thermal resistance in the following parts.

Fig. 3 shows temperature distribution along the $z$-direction in the channel of a pure $\mathrm{Cu}$, mono-, tri- and penta-layer graphene on a $\mathrm{Cu}$ surface obtained for the simulation procedure described in the previous section. The hot reservoir on the left was maintained at $360 \mathrm{~K}$, while the cold reservoir on the right was maintained at $280 \mathrm{~K}$. After reaching steady state in the presence of temperature difference in the system, the local temperature was varied as a linear function with respect to position in the bulk liquid region. The local temperature measurements undergo fluctuations at the near interface as a result of the very fine bin size chosen $(0.2 \AA$ in the $z$-direction) to define the local density distributions. Owing to the density oscillations and depletion layers, the limited number of liquid molecules occupied in several local bins induced fluctuation of temperature values $[18,46]$. These liquid temperatures cannot be utilized to define temperature jump at the interfaces. With a coarser bin size, the fluctuations should diminish as shown in Fig. 3. Therefore, interfacial temperature jumps were determined by extrapolating the linear temperature from middle of channel (black line) to the solid surfaces, which were indicated by black arrows in Fig. 3 .

We observed temperature jumps between solid and liquid domains due to the mismatch in phonon spectrum. There forms 

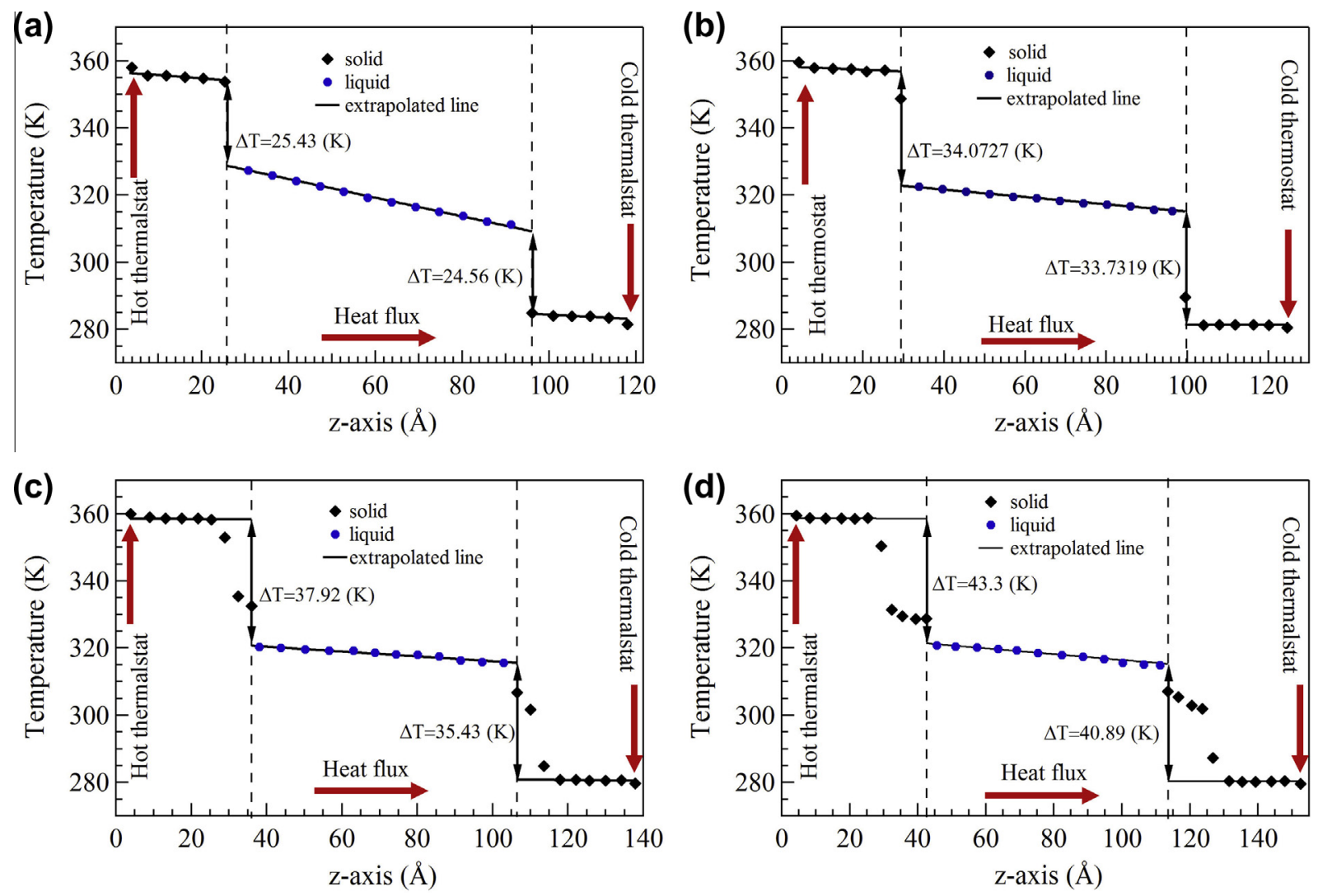

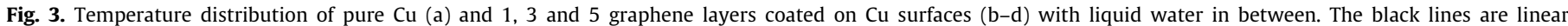
extrapolation lines of solid and liquid temperatures to the interfaces.

an additional temperature discontinuity at $\mathrm{Cu} /$ graphene interface with the addition of graphene coating on $\mathrm{Cu}$, indicating the effects of solid/solid interactions of different materials of nano-composite structure into the overall interfacial thermal resistance between solid surface and water. For simplicity, temperature jump was defined by extrapolating linear temperature from $\mathrm{Cu}$ substrates towards to solid/liquid interfaces across graphene layers. Thus, temperature jump calculations included both interface and nearinterface temperature mismatch of graphene/water and $\mathrm{Cu} /$ graphene regions, respectively [11]. Fig. 3 gives the temperature gradient within graphene domain as well. The temperature difference between the first graphene layer in contact with $\mathrm{Cu}$ were smaller than the difference with the rest of the graphene domains. This observation predicted that the thermal resistance forming between $\mathrm{Cu}$ and graphene is governed by the resistance at the region adjacent interface in addition to the interface itself [11]. Temperature jumps at both hot and cold solid walls were identical, indicating the negligible effects of wall temperature on interfacial thermal resistance.

To gain insight into the heat transfer mechanism across the interface between water and graphene-coated-Cu(111), we calculated the heat flux using Eq. (2), and utilized the temperature gradient $(\nabla T)$ of MD temperature profiles to determine the thermal conductivity $(\lambda)$ of confined water using Fourier's law $\vec{j}=-\lambda \nabla T$. For a given heat flux $\vec{j}$, the temperature jump can be associated with interfacial thermal resistance, which is a well-known called the Kapitza resistance $\left(R_{K}\right)$ and is described as $\Delta T=-R_{K} \vec{j} . \vec{n}$, where $\vec{n}$ is the outward unit normal from the wall. Simultaneously, the Kapitza length $\left(L_{K}\right)$ is defined as the thickness of the bulk medium extrapolated from the temperature profile of liquid to solid where the wall temperature is reached. The Kapitza length can be denoted as $\Delta T=\left.L_{K} \frac{\partial T}{\partial n}\right|_{\text {liquid }}$, where $\partial T / \partial n$ is the temperature gradient on the liquid side. Heat flux was calculated using Eq. (2) is shown in Fig. 4 (a), which was illustrated to decrease with an increase of the graphene coating layers. Fig. 4(b) shows the thermal conductivity predicted using Fourier's law, which yielded a constant value of $0.85 \mathrm{~W} / \mathrm{mK}$ at an average temperature of $320 \mathrm{~K}$. Therefore, the results supports the idea that the thermal resistance on the interface solely depends on the interfacial characteristics, and the thermal conductivity of the liquid is independent of the thermal resistance at the interface. This result was expected since the thermal conductivity is a material property, while the heat flux and temperature gradient depend on transport at the interface. In addition, liquid water pressure inside the channel was found to be $42.5 \mathrm{MPa}$ at the corresponding density of $1 \mathrm{~g} / \mathrm{cm}^{3}$ and temperature of $320 \mathrm{~K}$. Those of results are in a good agreement with liquid water properties at the similar conditions reported by Romer et al. [47]. Higher pressure and density than the initial setup values (see Section 2) were observed due to the reduction of liquid volume as a result of depletion layers formation within nanochannel. In principle, small variations in volume of confined liquid induce large deviations in density and pressure, which is characteristic of nanoscale systems (i.e., limited volume and finite number of molecules). This phenomenon was widely observed in computer simulations of fluid film confined between solid walls $[48,49]$. Increase of water pressure provides advantage of non-evaporable liquid in nanochannel which should be maintained within temperature range of simulation domain. Also, it was validated that the simulated pressure value has no effect on the calculated interface thermal resistance due to the high atomic packing factor of $\mathrm{Cu}$ 

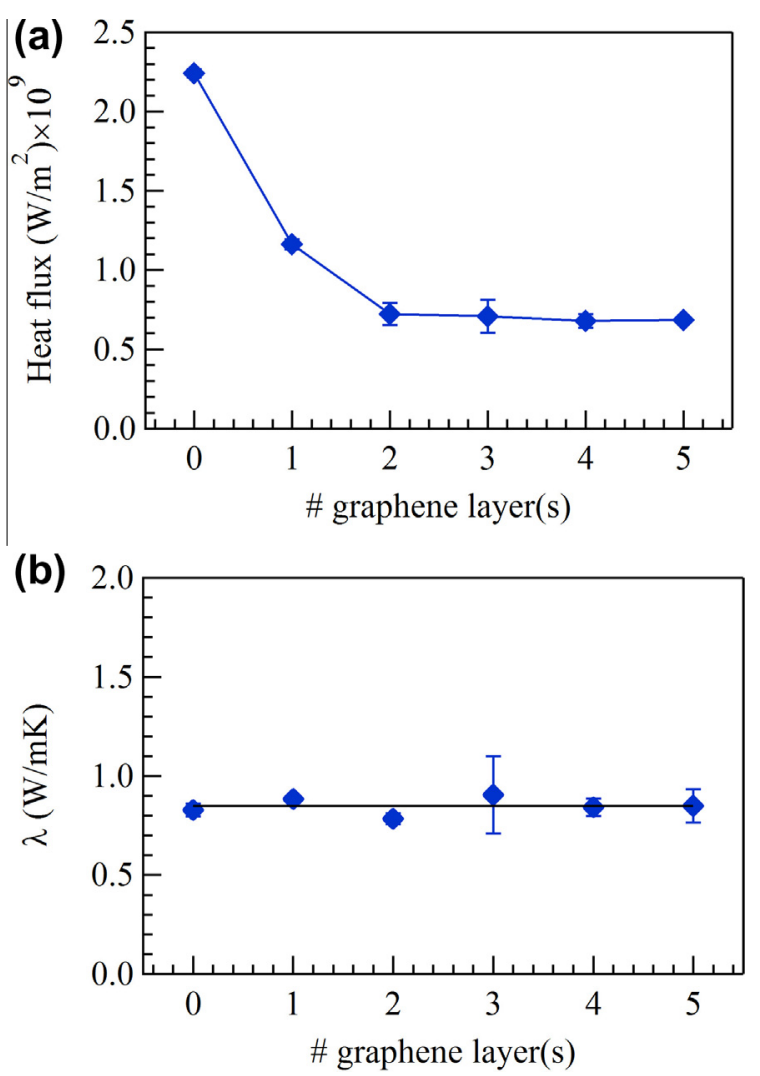

Fig. 4. Heat flux (a) and thermal conductivity (b) of liquid water calculated from Fourier's law with different number of graphene layers.

(111) and graphite surface which was investigated in detail in an earlier study [18]. Furthermore, the three-dimensional adsorption structure of liquid water on solid surfaces could be a key factor defining the thermal resistance at solid/liquid interfaces. However, such resolution requires further molecular studies which are beyond the scope of current research.

The variation of Kapitza length as a function of the number of graphene layers is shown in Fig. 5 where the addition of graphene layers on the $\mathrm{Cu}$ surface increases the Kapitza length values monotonically. The Kapitza length measured at the mono-layers of graphene-coated-Cu were approximately 2.5 times larger than the value at the bare $\mathrm{Cu}$ surface. Such behavior develops as a result

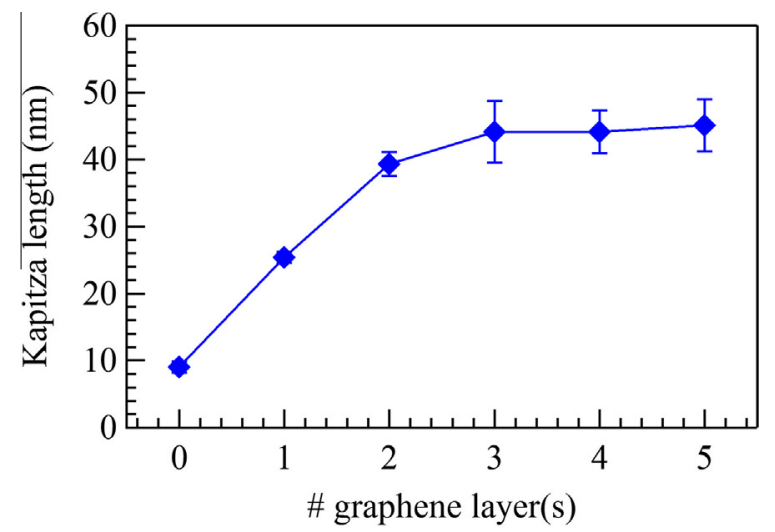

Fig. 5. Kapitza length variation as a function of graphene layers coated on the $\mathrm{Cu}$ (111) surface. The error bars reflect the average of several independent simulations for determining Kapitza length for each case. Some error bars are smaller than the symbols. of the translucent barrier of mono-layer graphene at the solid wall, and supports to an idea that graphene is only partial transparency [27,50,51]. These results are in contrast to the wetting transparency of graphene reported previously [25] which suggested that a single graphene layer allowed the significant transmission of $\mathrm{vdW}$ force from the solid wall to liquid water above the graphene. For interfaces that had more than two graphene layers, the thermal resistance gradually converged to the value measured between pure graphite and liquid water.

The calculated Kapitza resistance and Kapitza length values were in good agreement with the existing experimental measurements of the interfacial thermal resistance between the water and solids. In particular, Ge et al. [52] found a typical thermal conductance $\left(G_{K}=1 / R_{K}\right)$ at hydrophilic surfaces to be $G_{K}=100 \mathrm{MWm}^{-2} \mathrm{~K}^{-1}$ which corresponded to a Kapitza length of $L_{K}=6 \mathrm{~nm}$. On the other hand, an interfacial thermal conductance of $G_{K}=12 \mathrm{MWm}^{-2} \mathrm{~K}^{-1}$ [53] was observed at the interface of hydrophobic carbon nanotube suspended in liquid water, which yielded a significantly large Kapitza length of $L_{K}=50 \mathrm{~nm}$. Similar to these experimental measurements, our calculations of the interface thermal conductance shows variation over a large range of $18-88 \mathrm{MWm}^{-2} \mathrm{~K}^{-1}$ corresponding to a range of Kapitza length of $9-46 \mathrm{~nm}$.

\subsection{Effects of number of graphene layers to surface energy}

The broad range of interfacial thermal resistance obtained from hydrophilic to hydrophobic surface chemistries can be characterized associating with the effects of graphene layers on the surface energy between water and the nano-composite structure. The direct correlation between the surface energy per unit area with interfacial thermal resistance has been found in several previous studies using MD simulations [54,55]. In order to validate that correlation in our system, it was necessary to investigate how the graphene layer affected the surface energy per unit area of the nanocomposite structure with liquid water.

Adding graphene layers on $\mathrm{Cu}(111)$ directly impacted the surface energy of the nano-composite structure. The surface energy exerted on liquid water was formed due not only to water $/ \mathrm{Cu}$ interactions, but also water/graphene molecular force interactions depending on the number of graphene layers. Owing to the immense complexity to calculate the quantities of $\mathrm{Cu} /$ water and graphene/water energies separately within current simulation box, the surface energy is very sensitive to the penetration depth of energies in liquid region from both $\mathrm{Cu}$ and graphene, and to the local effects including liquid density fluctuations and water/ water interaction contributions into energy calculations in this region. The interfacial energies were calculated using mathematical expression for surface interactions energy between macroscopic objects based on its correlations with surface tension (or water contact angle on solid substrates) [56-58]. The fundamental goal of this modeling is to consider one material surface is coated by a very thin layer of other that changes their wetting properties with liquid water. In order to characterize the surface energy in such systems, a quantitative description of long-range vdW attraction and short-range repulsion between each pair of materials is required. This approach has successfully defined the surface energy of colloidal particles coated by polymers [59] or thin polystyrene films dewetting solid substrates [60].

The general expression of interfacial energy between two flat bodies is given as $\Phi(h)=c / h^{8}-A / 12 \pi h^{2}$ [57], in which the first term describes the strength of the short-range repulsive interactions with free parameter $c$, and the second term denotes a longrange attraction of vdW potential with the Hamaker constant $A$, while $h$ is the separation distance between two bodies. In our 
study, free parameters $c$ for water/Cu and water/graphite were reproduced by calculating surface tension energy via measuring microscopic water contact angle $(\theta)$ on bare $\mathrm{Cu}$, and 5 graphenecoated as $|\Phi(h)|=\gamma(1+\cos \theta)$, where $\gamma$ is surface tension of liquid water. For composited surface of graphene-coated on the $\mathrm{Cu}(111)$, the interaction energy can be approximated as [25]:

$$
\begin{aligned}
\Phi_{\text {total }}(h, d)= & \frac{c_{\mathrm{W} / \mathrm{Gr}}}{h^{8}}-\frac{c_{\mathrm{W} / \mathrm{Gr}}}{(h+d)^{8}}+\frac{c_{\mathrm{W} / \mathrm{Cu}}}{(h+d)^{8}}-\frac{A_{\mathrm{W} / \mathrm{Gr}}}{12 \pi h^{2}} \\
& +\frac{A_{\mathrm{W} / \mathrm{Gr}}}{12 \pi(h+d)^{2}}-\frac{A_{\mathrm{W} / \mathrm{Cu}}}{12 \pi(h+d)^{2}},
\end{aligned}
$$

where $h$ is the separation between water and graphene, $d$ is the thickness of the graphene film including the interlayer spacing between graphene and $\mathrm{Cu}$. Despite the well agreement of microscopic contact angle measurements on $\mathrm{Cu}\left(86.1^{\circ}\right)$ and 5 graphenecoated $\mathrm{Cu}$ substrate $\left(96.1^{\circ}\right)$ with experimental [25] and simulations [42] which yielded the values of $c$ as $c_{\mathrm{W} / \mathrm{Cu}}=2.482 \times 10^{-80} \mathrm{~J} \mathrm{~m}^{6}$ and $c_{\mathrm{W} / \mathrm{Cu}}=2.951 \times 10^{-81} \mathrm{~J} \mathrm{~m}^{6}$, the parameters $c$ further requires the fulfillment of macroscopic level, which cannot be obtained with a 2000 water molecules droplet of our MD simulations. Therefore, we adopted the $c$ values from Ref. [25] as $c_{\mathrm{W} / \mathrm{Cu}}=$ $2.52 \times 10^{-80} \mathrm{~J} \mathrm{~m}^{6}$ and $c_{\mathrm{W} / \mathrm{Gr}}=0.98 \times 10^{-80} \mathrm{~J} \mathrm{~m}^{6}$ which recovered the macroscopic contact angle of water on $\mathrm{Cu}\left(85.9^{\circ}\right)$ and graphite $\left(90.6^{\circ}\right)$ for our theoretical calculations. Eq. (5) is an expression for the interaction energy of the nano-composite structure depending on the thickness of graphene films coated on the $\mathrm{Cu}$. Eq. (5) also provides a characterization of the interfacial energy of our system from bare $\mathrm{Cu}$ to the graphite surface. Specially, it reduces to the interfacial energy of liquid water with bare $\mathrm{Cu}$ when $d=0$, and expands to pure graphite when $d$ approaches infinity. Furthermore, the quantitative energy contribution by each pair of interactions water/Cu and water/graphene to the total interfacial energy can be determined separately from Eq. (5). After reaching steady state in the presence of heat transfer, the equilibrium separation $h$ was found to be $1.83 \AA$ and $1.93 \AA$ at the interface of water with bare $\mathrm{Cu}$ and water with a graphene surface, respectively. Those values are found different from the ones reported by Rafiee et al. [25]. Different than ref [25] assuming frozen surfaces where the molecules are fixed in their corresponding crystal structures, we considered the interactions between $\mathrm{Cu}-\mathrm{Cu}$, graphene-graphene, and $\mathrm{Cu}$-graphene molecules in our MD simulations. Neglecting the thermal vibrations of solid surfaces may create computationally cheaper simulation; however, we validated in an earlier water/silicon wetting study [29] that the frozen/fixed surface systems developed unphysical behaviors. The interlayer spacing $d$ between the $\mathrm{Cu}$ surface and mono-layer graphene was measured as $2.9 \AA$, that values increased more $3.4 \AA$ when an additional alternating graphene layer was added.

Fig. 6 demonstrated the dependence of interfacial energy per unit area on the number of graphene layers. The value of the interfacial energy per unit area varied significantly when inserting mono-layer graphene between water and the bare $\mathrm{Cu}$ surface, indicating the strong effect of the graphene layer on the surface adsorption energy of the nano-composite structure with liquid water. This observation further supported the partially wetting transparency of graphene discussed in the previous section. The interfacial energy per unit area of the nano-composite structures converged to a value measured between graphite and water when more graphene layers were inserted on the $\mathrm{Cu}$ surface, reinforcing the water density structures described in Fig. 2 remain unchanged. The dependence of the surface energy of nano-composites structures on the number graphene layers was shown to be related to the interfacial thermal resistance as well. The interfacial thermal resistance has been found to be inversely proportional to the

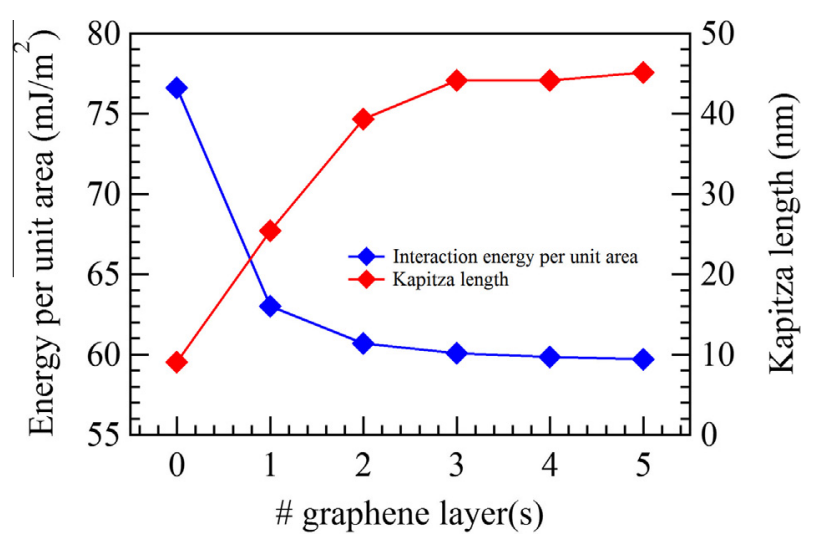

Fig. 6. Interfacial energy per unit area and Kapitza length as a function of graphene layer(s).

surface energy, which means the thermal resistance increased with a decrease in the surface energy $[23,54,55]$. Since the interaction strength coefficients between water/Cu and water/graphene were unchanged, the increase of number graphene layers led to the dominant surface interaction potential between the carbon atoms in graphene and liquid molecules rather than the contribution of solid $\mathrm{Cu}$ interactions with liquid to the entire surface energy. Thus, the solid surface interaction strength was predicted to vary from the strong surface adsorption of hydrophilic $\mathrm{Cu}$ to the weak surface adsorption of hydrophobic nano-composite structures with liquid water, thereby increasing Kapitza length at the interface with graphene-coated layers.

Eq. (5) not only characterizes the total adhesion energy between liquid water and nano-composite surface, but also allows the quantitative reconstruction of surface interaction energies of water/Cu and water/graphene separately. Fig. 7(bottom) illustrates the variations of the interaction energy between each pair of materials specifically in different cases. Once again, mono-layer graphene coated on the $\mathrm{Cu}$ broke down the wetting transparency properties of graphene by indicating that it was only $18 \%$ the interaction energy of the bare $\mathrm{Cu}$ transmitted across the graphene layer. It was also noted that the adsorption energy between water and mono-layer graphene was found to be $49.16 \mathrm{~mJ} / \mathrm{m}^{2}$ which is in agreement with the results observed both experimentally $\left(46.7 \mathrm{~mJ} / \mathrm{m}^{2}\right)$ [61], and mathematically $\left(49.1 \mathrm{~mJ} / \mathrm{m}^{2}\right)$ [62]. The adsorption energy between water and graphite converged to a value of $58.75 \mathrm{~mJ} / \mathrm{m}^{2}$ with five graphene layers coated on the $\mathrm{Cu}$ surface, which was similar to the experimental value of $54.8 \mathrm{~mJ} / \mathrm{m}^{2}$ [61], and the computational value of $59.9 \mathrm{~mJ} / \mathrm{m}^{2}$ [62], respectively. In addition, the surface energy calculated between the mono-layer graphene with water was approximately $16 \%$ less than that value observed with graphite with water, suggesting that the graphene sheet was more hydrophobic than the graphite sheet. A comparable observation is represented in several previous studies $[27,50,61,62]$.

It is hypothesized that the interaction strength between nanocomposite solid walls with liquid water is the integration of each molecular pair-wise interaction across the interface, which may be determined as:

$\varepsilon_{s l}=a \times \varepsilon_{\mathrm{Cu}-\mathrm{O}}+(1-a) \times \varepsilon_{\mathrm{C}-\mathrm{O}}$,

where $a$ is the percentage of the interaction energy per unit area of $\mathrm{Cu}$ surface with liquid water that is commensurate with the number of graphene layers on the Cu surface, and the rest is the contribution of the interaction energy of graphene with liquid water. In particular, the interaction strength of the nano-composite structure now 


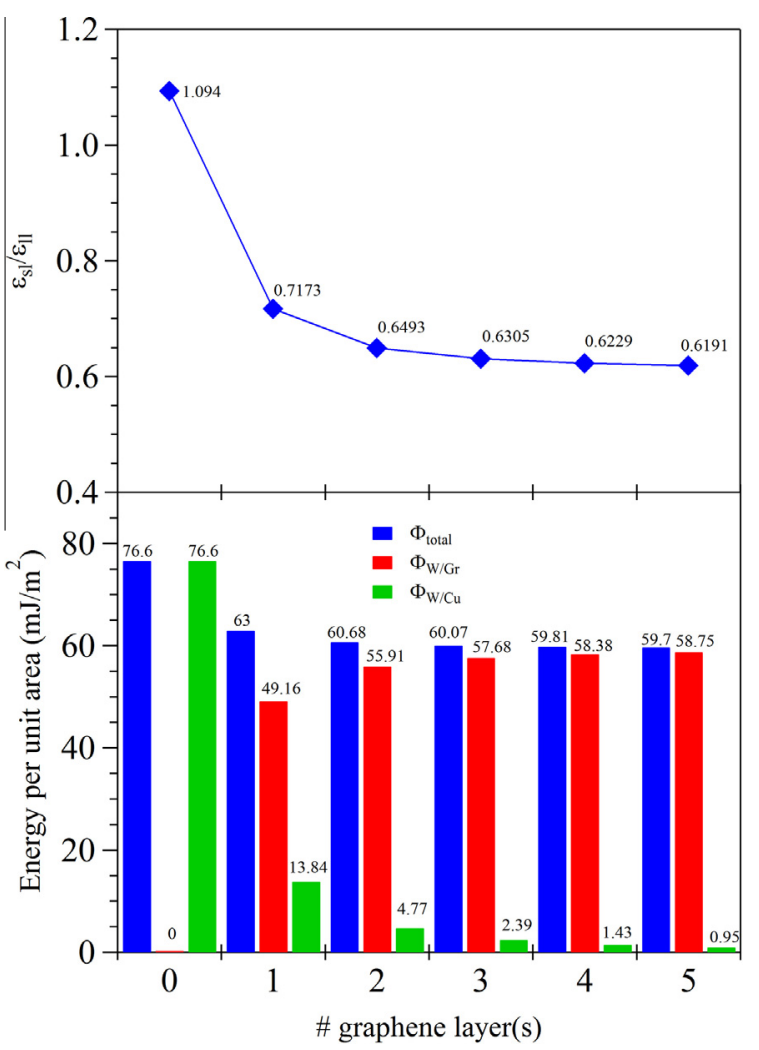

Fig. 7. (Bottom) Interfacial energy per unit area contributions of water/Cu and water/graphene as a function of the number of graphene layer(s). (Top) Interaction strength of nano-composite solid surfaces depends on the number of graphene layer(s).

varies within the range observed in liquid water with bare $\mathrm{Cu}$ to the value found with liquid water and bulk graphite which depends on the number of graphene layers on the $\mathrm{Cu}$ surface. The overall surface/liquid interaction strength values $\left(\varepsilon_{s l}\right)$ calculated by Eq. (6) were normalized with the well-known interaction potential value of water molecules $\left(\varepsilon_{l l}\right)$ to assist in systematically sorting the binding energy parameter for solid/liquid interaction as seen in previous studies [14-17]. Fig. 7(top) illustrates the variations of the solid/liquid interaction strength on the number of graphene layers coated on the $\mathrm{Cu}$ surface. The interaction strength parameters were derived from the surface energy per unit area of the nano-composite structure with different geometries of the subsurface regime of graphene. Therefore, they show behavior similar to that of the surface energy per unit area as indicated in Fig. 6.

The dependence of Kapitza length on surface wettability is used as the fundamental property characterizing heat transfer across the solid/liquid interface. Specifically, the exponential variation of the Kapitza length as a function of the surface wettability was shown for $\varepsilon_{s l} / \varepsilon_{l l} \leqslant 1$ in the studies of Xue et al. [15] and Kim et al. [16]. Those of studies established a comprehensive empirical heat transfer model based on the intermolecular interaction strength using MD simulations. Therefore, a similar approach used herein provides insight into the heat transfer mechanism across the interface of the nano-composite structure with liquid water. Fig. 8 reveals the variation of Kapitza length as a function of solid/liquid interaction strength, which depended on the thickness of graphene films. The use of curve fitting with the data allowed an approximation of the Kapitza length $L_{K} \propto \exp \left(\gamma \times \varepsilon_{s l} / \varepsilon_{l l}\right)$ within the range of $0.61 \leqslant \varepsilon_{s l} / \varepsilon_{l l} \leqslant 1.1$, with $\gamma=-3.436$. The coefficient $\gamma$ was found to be -1.9 in the study of Xue et al. [15], and -1.85 in the study of Kim et al. [16,17], respectively. The discrepancy of the

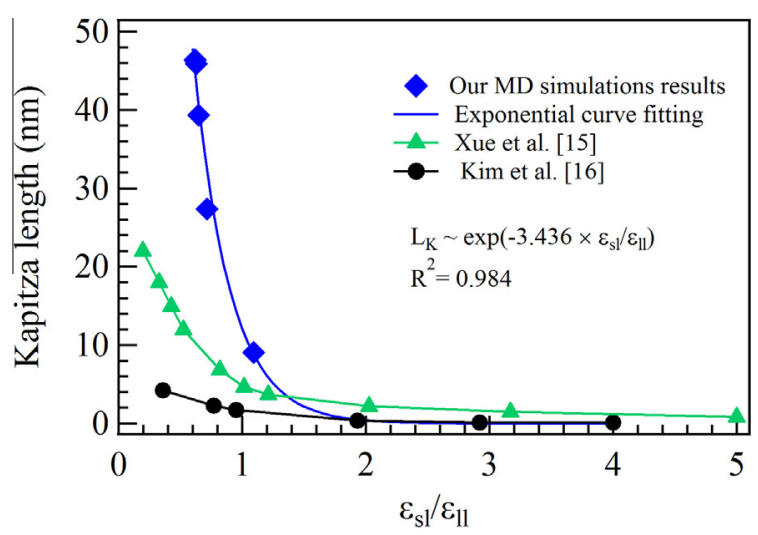

Fig. 8. Kapitza length as a function of the solid-liquid interaction strength parameter $\varepsilon_{s l} / \varepsilon_{l l} \cdot R^{2}=1$ corresponds to an exact fit.

present data with the aforementioned studies can be explained as the follows: (1) the atomic mass and size of both solid and liquid atoms were assume to be equal in prior studies, which is not practical given the reality of most materials; (2) the solid materials in the present study were modeled by well-known and accurate force fields of EAM and AIREBO potentials with respect to $\mathrm{Cu}$ and graphene. They included the potential energy of the electron density for $\mathrm{Cu}$ atoms or of the covalent bonds for carbon atoms which was far more complex and exhibited multiple degrees of freedom as opposed to the LJ interatomic potential [15] or simple massspring harmonic vibrations with a single degree of freedom [16,17]; (3) The solid surface consisted of nano-composite structures of graphene layers coated on $\mathrm{Cu}$, in which the solid/liquid interaction strength was the integration of both solid materials with liquid water, and showed a significant dependence on the thickness of the subsurface composites; i.e., the number of graphene layers; (4) The SPC/E liquid water model was described as effective pair potentials composed of $\mathrm{LJ}$ and Coulombic term that was more sophisticated than monoatomic species like liquid argon, and caused the deviations in the results. We expect that our finding will play a key role in the design of heat dissipation across nanocomposite/water interfaces in nanoscale.

\subsection{Effects of Cu/graphene interaction strength on Kapitza resistance}

To analyze the effects of the interaction strength between the $\mathrm{Cu}(111)$ surface and graphene on interfacial thermal resistance of nano-composite structures with liquid water, we performed a series of simulations in the system of single graphene-coated on $\mathrm{Cu}(111)$ at different $\varepsilon_{\mathrm{Cu} / \mathrm{C}}$, which were on the order of the value shown in Table $1\left(\varepsilon_{\mathrm{Cu} / \mathrm{C}}^{*}=0.02578 \mathrm{eV}\right)$ as $1,0.8,0.66,0.5,0.4,0.33$,

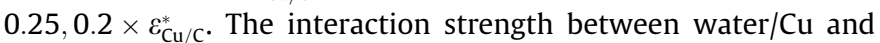
water/graphene was kept constant. The Kapitza length variation as a function of $\varepsilon_{\mathrm{Cu} / \mathrm{C}} / \varepsilon_{l l}$ is plotted in Fig. 9. We observed that Kapitza length separated into two different regimes at a critical value of $\varepsilon_{\mathrm{Cu} / \mathrm{C}} / \varepsilon_{l l}=1.6$. Regime 1 demonstrated a significant dependence of Kapitza length on the weak coupling of $\mathrm{Cu}$ and graphene. On the other hand, Kapitza resistance decreased slightly with strong coupling of $\mathrm{Cu}$ and graphene in regime 2 . The negligible change of Kapitza length throughout regime 2 indicated that it was a finite limit for the maximum value of $\varepsilon_{\mathrm{Cu} / \mathrm{C}} / \varepsilon_{l l}$, and that thermal resistance should persist under strong repulsive forces that prevent the overlap of molecules. Therefore, the dependence of Kapitza length on the parameter of $\varepsilon_{\mathrm{Cu} / \mathrm{C}} / \varepsilon_{l l}$ should not be utilized at extreme limits [15]. Interestingly, our results illustrated a notable increase of temperature jumps at the interlayer of $\mathrm{Cu}$ and graphene with weak atomic bonding that enlarged the Kapitza length 


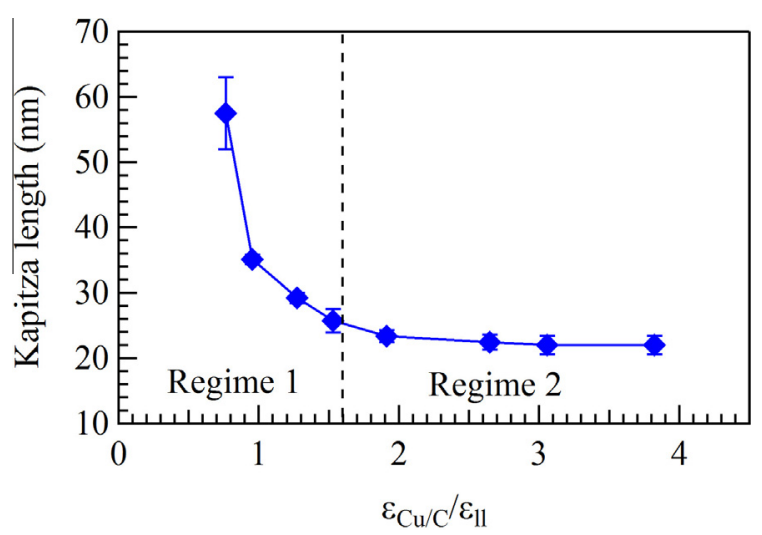

Fig. 9. Kapitza length variation as a function of interaction strength parameter $\varepsilon_{C u / C} / \varepsilon_{l l}$.The error bars reflects the average of several independent simulations for determining Kapitza length for each case.

at the interface of nano-composite structure with liquid water. At the same time, the interlayer spacing between $\mathrm{Cu}$ and graphene, and interface spacing between graphene with water remained unchanged. Therefore, the theoretical atomics force macroscopic approach, which depends on the thickness of subsurface graphene, cannot fully explain the surface energy and interaction strength of the nano-composite structure with different interaction strength values of $\mathrm{Cu}$ and graphene layers $\left(\varepsilon_{\mathrm{Cu} / \mathrm{C}} / \varepsilon_{l l}\right)$. Despite the limitations in characterizing the interaction strength of the nano-composite structure with different $\varepsilon_{\mathrm{Cu} / \mathrm{C}}$ values, our results still indicated the significant dependence of interfacial thermal resistance on the solid/solid interaction strength of the materials that were composed of $\mathrm{Cu}$ and graphene. An advanced model that completely defines the dependence of the strength of the nano-composite interaction with regards to solid/solid bonding strength will be the primary focus of future work.

\section{Conclusion}

We have systematically investigated the effects of the number of graphene layers on Kapitza length between the nanocomposite structures and water using non-equilibrium molecular dynamics. Our results revealed that the Kapitza length gradually increased with the increase of the number of graphene layers coated on the $\mathrm{Cu}$ surface. The measured monotonic decrease of the interfacial energy per unit area with the addition of graphene layers on the $\mathrm{Cu}$ surface explained the observed increase of the Kapitza length. The Kapitza length at the interface of the monolayer graphene and water was found 2.5 times larger than that value measured at the bare $\mathrm{Cu}$ surface. This result challenges the hypothesis of "transparency of graphene coating" introduced by the earlier researchers. The calculated surface energy of monolayer graphene also indicated that graphene transmits only $\sim 18 \%$ of the van der Waals energy of the Cu surface to the liquid domain, which reinforced the wetting translucency of graphene.

We further studied the corresponding contributions of the water/Cu and water/graphene interactions into the resulted interfacial energies of the each cases having different number of graphene layers. Using the strength values for water/Cu and water/graphene interactions, we calculated an overall interaction strength value for the nano-composites depending on the energy contribution of the each material forming the structure. Similar to our earlier investigations [16,17], the variation of the Kapitza length was found to be an exponential function of the estimated interaction strength values for nano-composite structures. Furthermore, the effect of $\mathrm{Cu} /$ graphene interactions on Kapitza length at the interface was also investigated. Change in Kapitza length was negligible in the case of strong coupling of $\mathrm{Cu} /$ graphene; however, it exhibited high thermal resistance with weak atomic bonding of $\mathrm{Cu}$ and graphene. Result offers a significant insight into the intermolecular interactions taking place at nano-composite surfaces, and provides an estimation of the interfacial thermal resistance between graphene-coated-metal surfaces and liquid water in heat transfer devices.

\section{Conflict of interest}

None.

\section{Acknowledgements}

This work was supported by the 2016 research fund of University of Ulsan, South Korea.

\section{References}

[1] K. Kim, From the future Si technology perspective: challenges and opportunities, IEEE Int. Electron Device Meeting (IEDM) 10 (2010) 111-119.

[2] K. Kim, J.Y. Choi, T. Kim, S.H. Cho, H.J. Chung, A role for graphene in siliconbased on semiconductor devices, Nature 479 (7373) (2011) 338-344.

[3] C. Lee, X. Wei, J.W. Kysar, J. Hone, Measurement of the elastic properties and intrinsic strength of monolayer graphene, Science 321 (5887) (2008) 385-388.

[4] K.I. Bolotin, K.J. Sikes, Z. Jiang, M. Klima, G. Fudenberg, J. Hone, P. Kim, H.L. Stormer, Ultrahigh electron mobility in suspended graphene, Solid State Commun. 146 (9-10) (2008) 351-355.

[5] K.S. Novoselov, A.K. Geim, S.V. Morozov, D. Jiang, Y. Zhang, S.V. Dubonos, I.V. Grigorieva, A.A. Firsov, Electric field effect in atomically thin carbon films, Science 306 (5696) (2004) 666-669.

[6] J.H. Seol, I. Jo, A.L. Moore, L. Lindsay, Z.H. Aitken, M.T. Pettes, X. Li, Z. Yao, R. Huang, D. Broido, N. Mingo, R.S. Ruoff, L. Shi, Two-dimensional phonon transport in supported graphene, Science 328 (5975) (2010) 213-216.

[7] J.M. MacLeod, F. Rosei, Molecular self-assembly on graphene, Small 10 (6) (2013) 1038-1049.

[8] S. Chen, L. Brown, M. Levendorf, W. Cai, S.Y. Ju, J. Edgeworth, X. Li, C.W. Magnuson, A. Velamakanni, R.D. Piner, J. Kang, J. Park, R.S. Ruoff, Oxidation resistance of graphene-coated $\mathrm{Cu}$ and $\mathrm{Cu} / \mathrm{Ni}$ alloy, ACS Nano 5 (2) (2011) $1321-1327$.

[9] G.L. Pollack, Kapitza resistance, Rev. Mod. Phys. 41 (1) (1969) 48-81.

[10] M. Shen, P.K. Schelling, P. Keblinski, Heat transfer mechanism across few-layer of graphene by molecular dynamics, Phys. Rev. B 88 (4) (2013) 045444-1045444-9.

[11] M. Hu, D. Poulikakos, Graphene mediated thermal resistance reduction at strongly coupled interfaces, Int. J. Heat Mass Transfer 62 (2013) 205-213.

[12] R. Mao, B.D. Kong, C. Gong, S. Xu, T. Jayasekera, K. Cho, K.W. Kim, Firstprinciples calculation of thermal transport in metal/graphene systems, Phys. Rev. B 87 (16) (2013) 165410-1-165410-7.

[13] S.W. Chang, A.K. Nair, M.J. Buehler, Geometry and temperature effects of the interfacial thermal conductance in copper- and nickel-graphene nanocomposites, J. Phys. Condens. Matter 24 (24) (2012) 245301-1-245301-6.

[14] J.L. Barrat, F. Chiaruttini, Kapitza resistance at the liquid-solid interface, Mol. Phys. 101 (11) (2003) 1605-1610.

[15] L. Xue, S.R. Phillpot, S.U.-S. Choi, J.A. Eastman, Two regimes of thermal resistance at a liquid-solid interface, J. Chem. Phys. 118 (1) (2003) 337-339.

[16] B.H. Kim, A. Beskok, T. Cagin, Molecular dynamics simulations of therma resistance at the liquid-solid interface, J. Chem. Phys. 129 (17) (2008) 1747011-174701-9.

[17] B.H. Kim, Thermal resistance at a liquid-solid interface dependent on the ratio of thermal oscillation frequencies, Chem. Phys. Lett. 554 (2012) 77-81.

[18] A. Pham, M. Barisik, B.H. Kim, Pressure dependence of Kapitza resistance at gold/water and silicon/water interfaces, J. Chem. Phys. 139 (24) (2013) 244702-1-244702-10.

[19] A.T. Pham, M. Barisik, B.H. Kim, Molecular dynamics simulations of Kapitza length for argon-silicon and water-silicon interfaces, Int. J. Precis. Eng. Manuf. 15 (2) (2014) 323-329.

[20] M. Barisik, A. Beskok, Temperature dependence of thermal resistance at the water/silicon interface, Int. J. Therm. Sci. 77 (2014) 47-54.

[21] Z. Shi, M. Barisik, A. Beskok, Molecular dynamics modeling of thermal resistance at argon-graphite and argon-silver interfaces, Int. J. Therm. Sci. 59 (2012) 29-37.

[22] M. Barisik, A. Beskok, Boundary treatment effects on molecular dynamics simulations of interface thermal resistance, J. Comp. Phys. 231 (23) (2012) $7881-7892$.

[23] H. Hu, Y. Sun, Effect of nanopatterns on Kapitza resistance on water-gold interface during boiling: a molecular dynamics study, J. Appl. Phys. 112 (5) (2012) 053508-1-053508-6. 
[24] M. Hu, J.V. Goicochea, B. Michel, D. Poulikaskos, Water nanoconfinement induced thermal enhancement at hydrophilic quarzt interfaces, Nano Lett. 10 (1) (2010) 279-285.

[25] J. Rafiee, X. Mi, H. Gullapalli, A.V. Thomas, F. Yavari, Y. Shi, P.M. Ajayan, N.A Koratkar, Wetting transparency of graphene, Nat. Mater. 11 (2012) 217-222.

[26] R. Raj, S.C. Maroo, E.N. Wang, Wettability of graphene, Nano Lett. 13 (4) (2013) $1509-1515$

[27] C.J. Shih, M.S. Strano, D. Blankschtein, Wetting translucency of graphene, Nat. Mater. 12 (2013) 866-869.

[28] M.P. Allen, D.J. Tildesley, Computer Simulation of Liquids, Oxford Science Publications Oxford University Press, 1989.

[29] M. Barisik, A. Beskok, Wetting characterization of silicon $(1,0,0)$ surface, Mol. Simul. 39 (9) (2013) 700-709.

[30] P.E. Hopkins, M. Baraket, E.V. Barnat, T.E. Beechem, S.P. Kearney, J.C. Duda, J.T. Robinson, S.G. Walton, Manipulating thermal conductance at metal-graphene contacts via chemical functionalization, Nano Lett. 12 (2) (2012) 590-595.

[31] L. Chen, S. Kumar, Thermal transport in graphene supported on copper, J. Appl. Phys. 112 (4) (2012) (043502-1-043502).

[32] Y. Guo, W. Guo, Structure transformation of partially confined copper nanowires inside defected carbon nanotubes, Nanotechnology 17 (18) (2006) 4726-4730.

[33] Z. Xu, M.J. Buehler, Nanoengineering heat transfer performance at carbon nanotube interfaces, ACS Nano 3 (9) (2009) (2767-2275).

[34] G. Giovannetti, P.A. Khomyakov, G. Brocks, V.M. Karpan, J. van den Brink, P.J. Kelly, Doping graphene with metal contacts, Phys. Rev. Lett. 101 (2) (2008) 026803-1-026803-4.

[35] L. Gao, J.R. Guest, N.P. Guisinger, Epitaxial graphene on Cu (111), Nano Lett. 10 (9) (2010) 3512-3516.

[36] Z. Xu, M.J. Buehler, Interface structure and mechanics between graphene and metal substrates: a first-principles study, J. Phys. Condens. Matter 22 (48) (2010) 485301-1-485301-5.

[37] H.J.C. Berendsen, J.R. Grigera, T.P.J. Straatsma, The missing term in effective pair potentials, J. Phys. Chem. 91 (24) (1987) 6269-6271.

[38] J.P. Ryckaert, G. Ciccotti, H.J.C. Berendsen, Numerical integration of the cartesian equations of motion of a system with constraints: molecular dynamics of $n$-alkanes, J. Comp. Phys. 23 (3) (1977) 327-341.

[39] J. Mei, J.W. Davenport, G.W. Fernando, Analytic embedded-atom potentials for fcc metals: application to liquid and solid copper, Phys. Rev. B 43 (6) (1991) 4653-4658.

[40] S.J. Stuart, A.B. Tutein, J.A. Harrison, A reactive potentials for hydrocarbons with intermolecular interactions, J. Chem. Phys. 112 (14) (2000) 6472-6486.

[41] J. Yu, J.G. Arma, Effects of short-range attraction in metal epitaxial growth, Phys. Rev. Lett. 89 (28) (2002) 286103-1-286103-4.

[42] T. Werder, J.H. Walther, R.L. Jaffe, T. Halicioglu, P. Koumoutsakos, On the water-carbon interactions for use in molecular dynamics simulations of graphite and carbon nanotubes, J. Phys. Chem. B 107 (2003) 1345-1352.
[43] J.H. Irving, J.G. Kirkwood, The statistical mechanical theory of transport processes. IV. The equations of hydrodynamics, J. Chem. Phys. 18 (1950) 817829.

[44] B.D. Todd, P.J. Daivis, D.J. Evans, Heat flux vector in highly inhomogeneous nonequilibrium fluids, Phys. Rev. E 51 (5) (1995) 4362-4368.

[45] S.J. Plimpton, J. Comput, Fast parallel algorithms for short-range molecular dynamics, J. Comput. Phys. 117 (1) (1995) 1-19.

[46] B.H. Kim, A. Beskok, T. Cagin, Viscos heating in nanoscale shear driven liquid flows, Microfluid. Nanofluid. 9 (2010) 31-40.

[47] F. Romer, A. Lervik, F. Bresme, Nonequilibrium molecular dynamics simulations of the thermal conductivity of water: a systematic investigation of the SPC/E and TIP4P/2005 models, J. Chem. Phys. 137 (7) (2012) 074503-1074503-8.

[48] P.A. Thompson, G.S. Grest, M.O. Robbins, Phase transition and universal dynamics in confined films, Phys. Rev. Lett. 68 (1992) 3448-3451.

[49] G. Nagy, M.C. Gordillo, E. Guardia, J. Marti, Liquid water confined in carbon nanochannels at high temperatures, J. Phys. Chem. B 111 (2007) 12524-12530.

[50] C.J. Shih, Q.H. Wang, S. Lin, K.C. Park, Z. Jin, M.S. Strano, D. Blankschtein, Breakdown in the wetting transparency of graphene, Phys. Rev. Lett. 109 (17) (2012) 176101-1-176101-5.

[51] Editorial, Not so transparent, Nat. Mater. 12 (2013) 865.

[52] Z. Ge, D.G. Cahill, P.V. Braun, Thermal conductance of hydrophilic and hydrophobic interfaces, Phys. Rev. Lett. 96 (18) (2006) 18610-1-186101-4.

[53] S.T. Huxtable, D.G. Cahill, S. Shenogin, L. Xue, R. Ozisik, P. Barone, M. Usrey, M. S. Strano, G. Siddons, M. Shim, P. Keblinski, Interfacial heat flow in carbon nanotube suspensions, Letters 2 (2003) 731-734.

[54] N. Shenogina, R. Godawat, P. Keblinski, S. Garde, How wetting and adhesion affect thermal conductance of a range of hydrophobic to hydrophilic aqueous interfaces, Phys. Rev. Lett. 102 (15) (2009) 156101-1-156101-4.

[55] Y. Wang, P. Keblinski, Role of wetting and nanoscale roughness on thermal conductance at liquid-solid interface, Appl. Phys. Lett. 99 (7) (2011) 073112 1-073112-3.

[56] J.N. Israelachvili, Intermolecular and Surface Forces, third ed., Academic Press, 2011.

[57] R. Seemann, S. Herminghaus, K. Jacobs, Dewetting patterns and molecular forces: a reconciliation, Phys. Rev. Lett. 84 (24) (2001) 5534-5537.

[58] R. Tadmor, The London-van der Waals interaction energy between objects of various geometries, J. Phys. Condens. Matter 13 (9) (2001) L195-L202.

[59] R. Tadmor, R.E. Rosensweig, J. Frey, J. Klein, Resolving the puzzle of ferrofluid dispersants, Langmuir 16 (24) (2000) 9117-9120.

[60] R. Seemann, S. Herminghaus, K. Jacobs, Gaining control of pattern formation of dewetting liquid films, J. Phys. Condens. Matter 13 (21) (2001) 4925-4938.

[61] S. Wang, Y. Zhang, N. Abidi, L. Cabrales, Wettability and surface free energy of graphene films, Langmuir 25 (18) (2009) 11078-11081.

[62] F. Taherian, V. Marcon, N.F.A. van der Vegt, What is the contact angle of water on graphene?, Langmuir 29 (2013) 1457-1465 